\title{
Conformational flexibility in protein molecules
}

\author{
from Robert Huber
}

IN describing protein molecules the term flexibility is used with quite different meanings. A precise definition for a particular protein molecule would require the determination of the number, energy and geometry of the various stable conformational states the molecule might have, the kinetic parameters of interconversion and the thermal motion of all the atoms in each conformer: a considerable task, requiring a great variety of experimental techniques, if feasible at all. Several rather different experimental approaches have provided evidence that protein molecules are indeed flexible: deuterium-hydrogen exchange under nondenaturing conditions ${ }^{1}$, fluorescence quenching of internal tryptophan residues by oxygen diffusing into the protein molecule ${ }^{2}$, ESR, NMR, and fluorescence depolarisation. These last spectroscopic techniques allow one to determine the mobility as a function of temperature of certain residues whose spectroscopic signal can be identified. The power of these methods is clearly demonstrated by recent experiments, using NMR techniques, which show that the aromatic rings of tyrosyl and phenylalanyl residues in pancreatic trypsin inhibitor (PTI) flip $^{3-5}$. Model calculations show that aromatic ring flipping is a consequence of substantial breathing motions of the protein, which include movement of the polypeptide backbone ${ }^{6,7}$. The mobility of tryptophanyl residues has been probed, using very short and intense light pulses to measure fluorescence depolarisation ${ }^{8}$. Motions in the picosecond time range are found, but the degree of flexibility is different for different protein species, some being rather rigid, some soft. Recent molecular dynamics calculations on PTI $^{9}$ show structural fluctuations in the picosecond range of a size in rough agreement with the observed crystallographic temperature factor ${ }^{10}$ and promise to provide the theoretical background to motions in proteins. However, the computational problems are enormous and, so far, allow the analysis of a small protein molecule such as PTI for a few picoseconds only. This makes extrapolation to equilibrium properties and comparison with the time-averaged crystal structures problematic, as does the neglect of solvent effects in calculations performed to date. More refined dynamics calculations are currently being pursued.

Protein crystallography as a means to gain information about flexibility is unique

Robert Huber is at the Max-Planck-Institut für Biochemie, Munich. in providing us with an overall view of the molecule in contrast to the spectroscopic techniques which focus on a particular label. However, there are a number of theoretical and experimental problems. As the physical diffraction process is instantaneous compared with lattice vibrations, the 'temperature' factor determined crystallographically does not differentiate between thermal motion and static disorder. Static disorder may have components from conformational microheterogeneity of the protein molecules (substates or microscopic states) and from lattice defects. The interesting parameters, thermal motion and substates are difficult to determine accurately and still more difficult to separate from one another. The experimental problem has been partly overcome since the development of techniques for refinement of protein crystal structures, some five years ago.

Protein structures were successfully refined ${ }^{10-12}$ using principles borrowed from small molecule crystallography, extensively modified and adapted to the much larger problem ${ }^{13}$. From these studies it was obvious that the 'temperature' factors of individual atoms were important variables for the correct description of the protein structure. However, the values were regarded with some suspicion, as they changed from atom to atom in a rather erratic way due to the limited resolution of the diffraction data. But, when the 'temperature' factors were averaged for rigid groups and smoothed along the main and side chains, physically reasonable features were obvious: 'temperature' factors increased in external polypeptide loops and external side chains were more mobile than internal ones.

\footnotetext{
1. Woodward \& Hilton A. Rev. Biophys. Bioeng. 8,99 (1979). 2. Lakowicz \& Weber Biochemistry 12, 417 (1973).

3. Snyder et at. Biochemistry 14, 3765 (1975).

4. Wagner et al. Biophys. Struct. Mechanism 2, 139 (1976).

5. Snyder et al. Biochemistry 15, 2275 (1976).

6. Gelin \& Karplus Proc. natn. Acad. Sci. U.S.A. 72, 2002 (1975).

. Hetzel et al. Biophys. Struct. Mechanism 2, 159 (1976).

8. Murro et at. Prac natn. Acad. Sci. U.S.A. 76, 56 (1979)

9. Karplus \& McCommon Nature 277, 578 (1979).

10. Deisenhofer \& Steigemann Acta Cryst. B31, 238 (1975).

11. Watenpaugh et al. Acta Cryst. B29, 943 (1973).

12. Huber et al. J. molec. Biol. 89, 73 (1974).

12. Huber et al. J. molec. Biol. 89, 73 (1974).

13. Diamond J. molec. Biot. 82, 371 (1974).

15. Ely et al. Biochemistry 17, 820 (1978).

16. Huber \& Bode Acc. Chem. Res. 11, 114 (1978).

17. Wiegand et al. Eur J. Biochem. 93, 41 (1978).

18. Bloomer et at. Nature 276, 362 (1978).

19. Stubbs et al. Nature 267, 216 (1977).

20. Harrison et al. Nature 276, 368 (1978).

21. Huber Trends in Biochem Sci. (in the press)

22. Jardetzky et al. Nature 273, 564 (1978)

23. Parak et al. Acta Cryst. A27, 573 (1971)

24. Epp et al. Biochemistry 14, 4943 (1975).

25. Steigemann \& Weber J. molec. Biol. 127, 309 (1979)
} 26. Richards J. molec, Biol, 82, 1 (1974).
Several examples of protein crystal structures are now known where disorder (either dynamic or static) is a dominating aspect: antibody molecules 14. 15. trypsinogen ${ }^{16}$, citrate synthase ${ }^{17}$, tobacco mosaic virus protein ${ }^{18,19}$ and tomato bushy stunt virus ${ }^{20}$. In these molecules substantial parts show no significant electron density indicating disorder in the crystalline state. In some of the molecular species rigid states are known with different functions, providing evidence for the functional significance of large scale disorder ${ }^{21}$. Except in the case of TMV protein, where NMR experiments have demonstrated increased thermal mobility of the disordered segments ${ }^{22}$, it is unclear which type of disorder (conformational heterogeneity or thermal motion) prevails in these examples. Lattice defects involving the molecule as a whole are excluded, of course, as most parts of the molecules are well ordered.

Two reports in this issue of Nature are centred on the question of conformational disorder and its static and dynamic components determined by crystallographic methods in two different systems: lysozyme (Artymiuk et al. page 563) and myoglobin (Frauenfelder et al. page 558). Two different lysozyme species crystallising in different crystal forms have been carefully refined and atomic 'temperature' factors determined. When these were averaged for the main chain atoms of each residue a close correspondence between the two crystal structures was obvious, suggesting that the variation in 'temperature' factor along the chain is a molecular property and not seriously influenced by crystal packing. A detailed mathematical analysis indicates a rigid body translation and libration of the molecule as a whole, overlaid with additional flexibility of some external loops. Among these are the loops determining the substrate binding cleft in lysozyme. A positive correlation between ordered secondary structure and 'rigidity', and between shielding of amino acid side chains and their 'mobility' is obvious. The slightly enhanced 'mobility' of the substrate binding area is intriguing, in particular, if it were to be found that these segments 'rigidify' on substrate binding; one wonders why nature utilises such small effects, having in mind the drastic segmental flexibility in the examples discussed before. Artymiuk et al. do not and cannot dissect the 'temperature' factors observed into their static and dynamic contributions. This problem is the main object of the paper by Frauenfelder $e t$ al. who analysed and carefully refined 
myoglobin crystals at various temperatures from 300 to $220 \mathrm{~K}$, with the ultimate goal of differentiating static and dynamic disorder by their low temperature limit. As protein crystals contain solvent which may not be allowed to freeze, the temperature range that is covered is small as is the effect on the 'temperature' parameters determined.

The principal result of Frauenfelder $e t$ $a l$,that 'temperature' factors are increased in the peripheral parts of myoglobin and correlate with the secondary structural elements, agrees with the observations on lysozyme and other proteins discussed. The authors also follow and compare the variation of the 'temperature' factors with temperature for internal and peripheral atoms: they make the novel and interesting observation that internal atoms 'freeze' more than peripheral ones. This leads the authors to the view that conformational heterogeneity (substates) is a dominent type of disorder, at least for the outer parts of myoglobin. As the temperature range covered is small and the effects measured marginally above the level of significance, it would be highly desirable to back up these results by going to substantially lower temperatures, perhaps using the shock-freezing technique developed by Parak et al. ${ }^{23}, 24$. These authors had shown by Mössbauer absorption spectroscopy on myoglobin crystals that about half of the crystallographic 'temperature' factor is due to static disorder. Mössbauer absorption measurements are insensitive to static disorder as long as the equilibration between different conformers is slow compared with the life time of the excited Mössbauer nucleus. The question of whether a protein molecule is to be regarded as a vibrating aperiodic crystalline solid or a 'semiliquid' 'glass', characterised by a larger number of substates of similar energy, is a topic of lively discussion at present. Of course, there is clear evidence for alternative side chain conformations in protein crystals (see for instance Epp et al. ${ }^{24}$ who observed conformational heterogeneity of an internal cysteine residue; or His $\mathbf{E} 7$ in erythrocruorin (Steigemann \& Weber $^{25}$ )). However, the uneasy feeling of a protein crystallographer about the 'semiliquid', 'glassy' protein picture comes from the fact that proteins are tightly packed ${ }^{26}$, multiply linked and cross-linked molecules which may have problems finding efficient alternative packing except, of course, for residues and loops interacting with solvent. It would, perhaps, be appropriate to defer these arguments until it is possible to define more precisely the extent to which substates exist in a particular type of disorder. The differentiation between a single vibrating conformer and an assemblage of a huge number of closely related substates separated by low energy barriers and in rapid equilibration becomes a matter of semantics, except close to $0 \mathrm{~K}$, a rather unphysiological temperature.

\section{Meteoroid fragmentation in the}

\section{auroral zones}

\section{from David W. Hughes}

Satellite measurements of the micrometeoroid dust particles in the Earth's vicinity have produced many interesting results in the last two decades. One of the more fascinating observations has come from experiment S 215 on the HEOS-2 spacecraft. Not only did this experiment measure the normal sporadic flux of these particles but it also found groups and swarms of micrometeoroids which had apparently been produced as lunar ejecta, and also as the debris from the electrostatic disruption of larger meteoroids.

H. Fechtig, E. Grün and G. Morfill of the Max-Planck-Institute for Nuclear Physics, Heidelberg, have produced a detailed analysis of these results in a recent edition of Planetary and Space Science (27, $511 ; 1979)$. We can dismiss the sporadic particles fairly quickly. They arrive randomly, (as expected) the number observed per unit time obeys Poisson statistics (as expected) and the flux is what one would expect from the zodiacal dust cloud, allowing for the enhancement produced by the gravitational field of the Earth. reach the satellite, by which time it will have spread out in space as a result of the velocity dispersion. Reasonable assumptions show that it will take about $7 \mathrm{~h}$ to sweep past the spacecraft. The experiment was working between 7 February, 1972 and 2 August, 1974 and during this time 19 group bursts were seen, individual groups taking between $40 \mathrm{~min}$ and $13 \mathrm{~h}$ to pass the spacecraft. Fechtig, Grün and Morfill tried to correlate the observation times of the groups with the occurrence of seismic events on the Moon recorded by apparatus left behind by the Apollo astronauts. There were ten times more seismic events than group events. This fact is not too disheartening, however, for fast ejecta would leave the impact point at an angle of less than $10^{\circ}$ to the surface, so only the lunar rim regions can provide ejecta detectable at the spacecraft.

The source suggested for the swarm bursts is the Earth's auroral zones. About $30 \%$ of the meteoroids in space with masses above $100 \mathrm{~g}$ have densities less than $1 \mathrm{~g}$ $\mathrm{cm}^{-3}$, and these meteoroids are thought to be fragile, crumbly, loose conglomerate bodies made up of collections of individual particles in the micron and even submicron range. In interplanetary space meteoroids are positively charged with potentials in the range from +3 to $+20 \mathrm{~V}$. The main cause of this is the photoelectric effect. Solar quanta of sufficient energy (more than about $10 \mathrm{eV}$ ) may dislodge electrons from the surface layers of the meteoroid and sufficient numbers of these electrons escape the Coulombic back-attraction to leave the meteoroid positively charged. This charge is somewhat reduced by the capture of solar wind electrons and there are other much less dominant effects such as proton capture, secondary electron emission, sputtering and thermionic emission that can be neglected.

However when this meteoroid enters the ambient plasma of the Earth's magnetosphere at an altitude of about $60,000 \mathrm{~km}$ it quickly becomes negatively charged. The dominant charging process here is collection of electrons and protons from the Maxwellian plasma. Remember that the thermal electron and proton velocities are much greater than the meteoroid velocity. The majority of electrons and protons that hit the meteoroid stick to it. Also a meteoroid of radius greater than $10^{-5} \mathrm{~cm}$ can completely stop electrons of energies less than $10 \mathrm{keV}$ and protons of energies less than $70 \mathrm{keV}$. S.P. Wyatt (Planet. Space Sci. 17, 155; 1969) estimates that an iron, nickel or silicate meteoroid in sunlight at the Earth's orbit would be losing about $2.5 \times 10^{10}$ electrons per second per $\mathrm{cm}^{2}$ due to the photoelectric effect, so this has to be taken into account too.

Fechtig, Grün and Morfill consider two very dissimilar regions in the magnetosphere - first, the plasmasheet, a region outside the dipolar field regime. Here the electron number densities are 